\title{
Potential to improve outcomes in surgery using Six Sigma
}

\author{
Parul J. Shukla, MBBS, MS, FRCS, FACRSI (Hon.) $)^{1}$, Savio G. Barreto MBBS, MS, PhD ${ }^{2}$ \\ 1 In Charge, Gastrointestinal and Hepato-Pancreato-Biliary Surgery Service, Tata Memorial Hospital, Mumbai, India. \\ 2 Modbury Hospital, South Australia, Australia
}

Key words: Six Sigma; Surgery; Outcome.

\section{Introduction}

The practice of surgery has undergone a paradigm shift over the past few decades from the performance of routine surgical procedures, to developing newer and safer surgical techniques, to the current state of refining skills and striving towards outcome-improvement. This transformation of surgery has not been limited only to surgical procedures but more recently to the conduct of surgical care and training.

While on the face of it, the new focus on improving outcomes in surgery and improving surgical outcomes appear one and the same, this is not entirely true!

The practice of surgery has multiple facets. An obviously important one is the performance of surgery. What is equally important is the training of surgeons. Addressing these two factors will go a long way improving surgical outcomes. However, the 'practice of surgery' does not end with these two facets. The realisation that the surgical care of the patients begins when the patient first presents to the clinic and ends only when they are discharged from the care of the surgical unit brings to the fore numerous intermediate points at which the practice of surgery and its outcomes needs attention. If the practice of surgery needs to advance to the next level, then attention needs to be directed to each of these individual facets.

Correspondence: Parul J. Shukla, In Charge, Gastrointestinal and Hepato-Pancreato-Biliary Surgery Service, Tata Memorial Hospital, Mumbai, India.

Email: pjshukla@doctors.org.uk

The Sri Lanka Journal of Surgery 2011;29(1):1-3. practice of surgery and its outcomes needs attention. If the practice of surgery needs to advance to the next level, then attention needs to be directed to each of these individual facets.

The medical profession has been frequently compared to the aviation industry - the major reason for this being, the need for precision or flawless execution of function. In the modern practice of medicine, as in aviation, harsh as it may seem, there is a growing perception that there is no room for error.

It is for this reason that the recent years have witnessed a major emphasis on process improvement. Principal surgical bodies around the world are attempting to develop quality surgical programs $[1,2]$. There is a major thrust towards the practice of evidence-based medicine, standardisation of surgical training, the development of surgical simulation training [3], the encouragement of the conduct and review of surgical audits within the practice of individual surgeons, as well as, surgical departments, and establishment of protocols to stream-line practice and encourage the use of the 'checklist' to reduce the likelihood of human error. These are steps in the right direction.

In the context of improving outcomes, instead of attempting to 'reinvent the wheel', it appears opportune that the surgical fraternity should turn to institutions / industries that have historically prided themselves on process improvement with an aim to adopt the strategies employed by them. One such highly effective 'process improvement' tool is the Six Sigma methodology. The Six Sigma methodology, introduced by Motorola and subsequently popularized by Jack Welch and General Electric (GE) in the 1990s, [4] is a 
'process excellence' tool, the target of which is continuous improvement. It achieves this by providing an organized process for the structured analysis of data based on a five-step problemsolving methodology. The primary steps in the execution of the tool revolve around the acronym DMAIC which stands for: Define or Develop a vision, Measure (collect baseline data on defects and possible causes), Analyse (create a focused problem statement), Improve (create possible solutions for root causes), and Control (develop and document standard practices)[5].

Central to the practice of Six Sigma methodology is the team that comprises persons who are a part of the system and who are knowledgeable about the situation being studied. They are guided by a specialist trained in the practice of Six Sigma methodology, referred to as a 'Six Sigma Black Belt'. The 'Black Belt' advises and aids the team in every step of the Six Sigma process. The baseline or historical data is compared with data obtained after the implementation of Six Sigma-driven changes in order to determine if desired changes in performance have been achievedhttp://www.ncbi.nlm.nih.gov/pubmed/1 2800564[6].

The concept of Six Sigma is not novel to health care or surgery. One of the first applications of this tool in health care was its successful use in reducing catheter-related bloodstream infections in an intensive care unit [7]. Since then others have used this tool to reduce turn around times between general surgical cases [8] and to improve perioperative outcomes in colorectal surgery[9], amongst other applications [10,11].

We have previously outlined numerous potential applications for the use of Six Sigma to improve outcomes in hepato-pancreato-biliary (HPB) surgery and HPB surgical training [5], providing clear principles on how the process improvement tool of Six Sigma could be adopted to improve outcomes in surgery, as a whole.

The introduction of the surgical simulation training affords another perfect scenario where Six Sigma could be applied. For instance, by identifying critical steps in the performance of certain complex surgical procedures, more focus could be directed on this step at the time of training on the simulator.
SixSigma could also be used to identify key areas that influence the functioning of surgical units when developing flow charts and protocols. And as pointed out earlier, the change in process could then be objectively compared with the previous outcomes to assess the benefit of a particular intervention.

If we are to strive towards the flawless execution of the practice of surgery, we need to define and measure the problem, analyse and then improve the situation with an aim to achieving a standard of practice or control in short, Six Sigma can help.

\section{References}

1. Cohen ME, Bilimoria KY, Ko CY, Hall BL. Development of an American College of Surgeons National Surgery Quality Improvement Program: morbidity and mortality risk calculator for colorectal surgery. J Am Coll Surg. 2009 ;208(6):1009-16.

2. Ingraham AM, Richards KE, Hall BL, Ko CY. Quality improvement in surgery: the American College of Surgeons National Surgical Quality Improvement Program approach. Adv Surg.44:251-67.

3. Sturm LP, Windsor JA, Cosman PH, Cregan P, Hewett PJ, Maddern GJ. A systematic review of skills transfer after surgical simulation training. Ann Surg. 2008;248(2):166-79.

4. Snee R. Six Sigma: the evolution of 100 years of business improvement methodology. Int J Six Sigma Competitive Advantage. 2004;1:4-20.

5. Shukla PJ, Barreto SG. Can we apply the process improvement tool Six Sigma to e $\mathrm{nh}$ a $\mathrm{nce}$ o u t c o m e s i n hepatopancreatobiliary surgery? HPB (Oxford). 2009;11(2):93-5.

6. Benedetto A. Six Sigma: not for the faint of heart. Radiol Manage. 2003;25:40-53.

7. Frankel HL, Crede WB, Topal JE, Roumanis SA, Devlin MW, Foley AB. Use of corporate Six Sigma performanceimprovement strategies to reduce incidence of catheter-related bloodstream infections in a surgical ICU. J Am Coll Surg. 2005;201(3):349-58. 
8. Adams R, Warner P, Hubbard B, Goulding T. Decreasing turnaround time between general surgery cases: a six sigma initiative. J Nurs Adm. 2004;34(3):140-8.

9. Shukla PJ, Barreto SG, Nadkarni MS. Application of Six Sigma towards improving surgical outcomes . Hepatogastroenterology. 2008;55(8283):311-4.

10. Cavagna E, Berletti R, Schiavon F, Scarsi B,
Barbato G. Optimized delivery radiological reports: applying Six Sigma methodology to a radiology department. Radiol Med. 2003;105(3):205-14.

11. Parker BM, Henderson JM, Vitagliano S, Nair BG, Petre J, Maurer WG, et al. Six sigma methodology can be used to improve adherence for antibiotic prophylaxis in patients undergoing noncardiac surgery. Anesth Analg. 2007;104(1):140-6. 